\title{
STABILITY AGAINST RADIAL PERTURBATIONS OF SLOWLY ROTATING NEUTRON STARS*
}

Kenzo ARAI and Keisuke KAMINISHI

Department of Physics, Kumamoto University

The dynamical equations governing pulsation in rotating neutron stars are derived in the framework of general relativity. Stellar models are constructed by using a realistic equation of state for cold neutron matter. Small radial displacement and slow rotation are treated as perturbations on spherically symmetric body. In these models the maximum masses are $1.761 \mathrm{M}_{\odot}$ at the central density $3.461 \times 10^{15} \mathrm{~g} \mathrm{~cm}^{-3}$ for a sequence of nonrotating configurations and $2.165 \mathrm{M}_{\odot}$ for rotating models with the critical angular velocity $\left(G M / R^{3}\right)^{1 / 2}$.

We examine the stability of the three lowest quasi-radial modes in these models. The characteristic time scale of radial pulsation is of oder $1 \mathrm{~ms}$ for stable neutron stars. Effects of rotation do not stabilize the equilibrium configurations, but enhance the instability. This result presents a striking contrast to the case of the Tooper polytrope calculated by Hartle et al. (1975). The $n=3 / 2$ polytropic equation has the value of the adiabatic index $T=5 / 3$, so that the constructed model can sustain its stability upto higher central densities where the relativistic effects become significant. The centrifugal force may contribute to the restoring one on a radial perturbation, because there exist the stable regions in the middle and outer layers of their model. On the other hand, for the realistic equation of state used here, we have $\Gamma \cong 0.4$ at the neutron drip point. This small value of $\Gamma$ makes the model unstable. In the models with higher central density the unstable outermost layer may be blown up by the centrifugal force and/or deformed significantly by the coriolis force. The fundamental mode is broken away rapidly as the angular velocity increases.

* This paper was presented by K. Kaminishi. 